\title{
SNAKES WITH TANGENT-BASED CONTROL AND ENERGIES FOR BIOIMAGE ANALYSIS
}

\author{
Virginie Uhlmann ${ }^{\star}$, Ricard Delgado-Gonzalo ${ }^{\star}$, and Michael Unser ${ }^{\star}$ \\ *Biomedical Imaging Group, École polytechnique fédérale de Lausanne (EPFL), Switzerland
}

\begin{abstract}
We propose a novel active contour for the analysis of filament-like structures and boundaries-features that traditional snakes based on closed curves have difficulties to delineate. Our method relies on a parametric representation of an open curve involving Hermite-spline basis functions. This allows us to impose constraints both on the contour and on its derivatives. The proposed parameterization enables tangential controls and facilitates the design of an energy term that considers oriented features. In this way, our technique can be used to detect edges as well as ridges. The use of the Hermite-spline basis is well suited to a semi-interactive implementation. We developed an ImageJ plugin, and present experimental results on real biological data.
\end{abstract}

Index Terms - Active contours, 2D, parameterization, microscopy, Hermite splines, steerable filters, orientation, segmentation, feature detection.

\section{INTRODUCTION}

Active contours [1], usually called snakes, are efficient tools for the automatic analysis of biomedical images (e.g., segmentation). Snakes consist of a curve that evolves within the image from an arbitrary user-defined initial position by optimizing a cost functional [2]. This cost function is problem-dependent and usually referred to as snake energy. Several types of snakes $[3,4,5]$ as well as different formulations for the energy term $[6,7]$ have been proposed.

In this paper, we introduce a novel type of active contour for the analysis of bio-images. Our snake is represented in terms of Hermite-spline basis functions. This framework extends the snake model based on B-splines $[8,6,9]$ by incorporating tangential controls (see Figure 1). Expressing the tangents of the contour explicitly in the parametric model has several advantages: it allows one to define an open-ended curve with natural extremities, and also gives accurate control over the snake in a Bézier-like fashion. We use a basis composed of two generating functions to represent the contour and its tangent field. The explicit parameterization of the tangent field allow us to define an energy functional based on steerable filters. Depending on the type of detectors, this energy can be tuned to be attracted either to edges or to ridges.

Our active contour is controlled using a reduced number of parameters (i.e., control points and tangent vectors). We designed a user-friendly interface that allows the user to interact with the snake. The contour can thereby be easily edited. This functionality is extremely valuable in complex images where the algorithm might need external feedback. Following our willingness to provide useful tools for the bioimage analysis community, the proposed algorithm

This work was funded by the Swiss SystemsX.ch initiative under Grant 2008/005 and the Swiss National Science Foundation under Grants 200020144355 and 200020-121763.

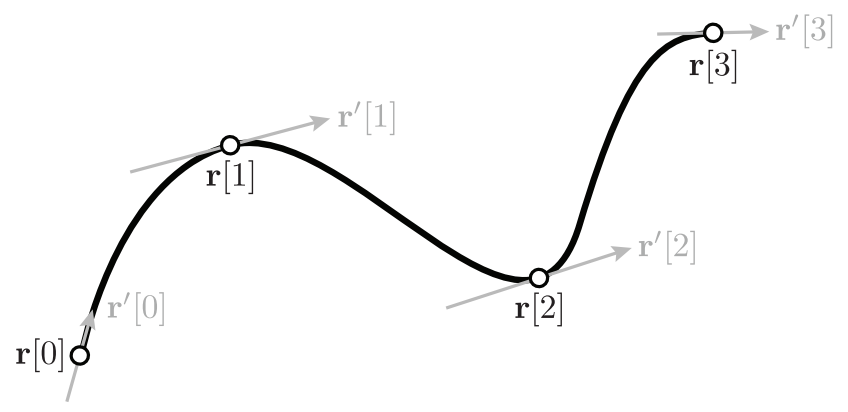

Fig. 1. Parametric representation of the Hermite snake. The vectors $\mathbf{r}[k]$ represent the anchor points and the $\mathbf{r}^{\prime}[k]$ their tangent controls. The tangent of the curve at each anchor point is depicted as a gray arrow.

is implemented as an open-source plugin for the free software ImageJ [10]. Finally, we demonstrate the efficiency of our algorithm with two examples of bioimage analysis.

\section{HERMITE SNAKE}

\subsection{Snake Model}

Most parametric snakes are defined as closed curves [2, 9]. Comparatively, there are much fewer solutions that can handle open curves $[11,12]$. Here, we introduce an active contour that is openended and fully defined by the values of few anchor points and their tangents. Our snake uses two complementary interpolation functions that provide both point-wise and tangential control. This is achieved by a mechanism commonly known as Hermite interpolation [13]. Therefore, we refer to our active contour model as the Hermite snake.

The parametric description of our Hermite snake is

$$
\begin{aligned}
\mathbf{r}(t)=\sum_{k=0}^{M-1} \mathbf{r}[k] \phi_{1}((M-1) t-k) \\
+\mathbf{r}^{\prime}[k] \phi_{2}((M-1) t-k),
\end{aligned}
$$

where $t \in[0,1]$. The positive integer value $M$ determines the number of anchor points and tangent vectors. The latter are defined by

$$
\begin{aligned}
\mathbf{r}[k] & =\left.\mathbf{r}(t)\right|_{t=\frac{k}{M-1}}, \\
\mathbf{r}^{\prime}[k] & =\left.\frac{\mathrm{d} \mathbf{r}}{\mathrm{d} t}(t)\right|_{t=\frac{k}{M-1}},
\end{aligned}
$$


respectively. We illustrate in Figure 1 the way in which these parameters determine the shape of a curve.

The fundamental ingredients of our model are two Hermite Bspline basis functions [14], which are given by

$$
\begin{gathered}
\phi_{1}(t)= \begin{cases}(2|t|+1)(|t|-1)^{2} & \text { for } 0 \leq|t| \leq 1 \\
0 & \text { for }|t|>1,\end{cases} \\
\phi_{2}(t)= \begin{cases}t(|t|-1)^{2} & \text { for } 0 \leq|t| \leq 1 \\
0 & \text { for }|t|>1 .\end{cases}
\end{gathered}
$$

The function $\phi_{1}$ interpolates the point values, while the derivative of $\phi_{2}$ interpolates the tangent values. The support of these two functions is within the range $[-1,1]$. The properties of the basis functions $\phi_{1}$ and $\phi_{2}$ that make this possible are

$$
\begin{array}{cl}
\phi_{1}(k)=\delta_{k}, & \phi_{1}^{\prime}(k)=0, \\
\phi_{2}(k)=0, & \phi_{2}^{\prime}(k)=\delta_{k},
\end{array}
$$

for integer values of $k$.

The proposed model gives direct control over tangents of the curve, which is desirable in many applications. For instance, when dealing with open curves, we can easily impose natural conditions on the extremities through the control of the tangent vectors. The fact that we are considering tangents of the curve is reminiscent of a Bézier parameterization. However, while Bézier curves are constructed with local bases and demand piece-wise interpolation, our Hermite snake benefits from a more global approach where the smoothness at the joining points is automatically enforced through (1).

\subsection{Feature Detection}

Once initialized, active contours are optimized by minimizing an energy functional. The definition of the energy functional depends on the image-analysis problem. Therefore, the quality of the segmentation strongly relies on the appropriate design of this term. Often, traditional snakes use edge-based detectors to guide them and are thus inefficient to detect ridge-like structures. We address this limitation and propose an energy term using steerable filters [15].

The optimal orientation $\hat{\boldsymbol{\theta}}_{\mathrm{opt}}$ and response $\rho_{\text {opt }}$ of the filter at a given location $\mathbf{x}=(x, y)$ on the image are given by

$$
\begin{aligned}
\hat{\boldsymbol{\theta}}_{\mathrm{opt}}(\mathbf{x}) & =\underset{\hat{\boldsymbol{\theta}}}{\operatorname{argmax}}\left(f(\mathbf{x}) * h\left(\mathbf{R}_{\hat{\boldsymbol{\theta}}} \mathbf{x}\right)\right) \\
\rho_{\mathrm{opt}}(\mathbf{x}) & =f(\mathbf{x}) * h\left(\mathbf{R}_{\hat{\boldsymbol{\theta}}_{\mathrm{opt}}} \mathbf{x}\right)
\end{aligned}
$$

The function

$$
h(\mathbf{x})=\sum_{k=1}^{N} \sum_{i=0}^{k} a_{k, i} \frac{\partial^{k-i}}{\partial x^{k-i}} \frac{\partial^{i}}{\partial y^{i}} g(\mathbf{x})
$$

is the detection template that is tuned to a given type of feature; $g$ is a Gaussian window, and $N$ is the order of the detector. Odd values of $N$ yield edge detectors, while even values generate ridge detectors. Filters built with larger values of $N$ have a higher SNR and better localization capabilities [15]. The filter can therefore be easily adapted to the kind and roughness of structure of interest by simply acting on this design parameter. In our implementation, we chose to use $N=\{1,3\}$ to detect edges and $N=\{2,4\}$ for ridges, depending on the desired level of precision.

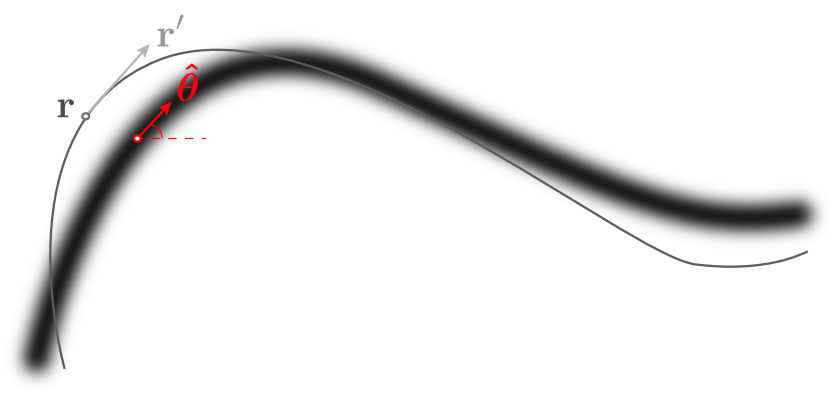

Fig. 2. Schematic representation of the Hermite snake $\mathbf{r}$, of its derivative in a point $\mathbf{r}^{\prime}$, and of its interaction with an object consisting of a dark ridge. The unit vector $\hat{\boldsymbol{\theta}}$ is the best response of the steerable filter for ridge detection. Since an estimation of $\hat{\boldsymbol{\theta}}$ is available for every point of the image, the inner product featured in the snake energy therefore favors tangents aligned to the orientation of best response.

\subsection{Image Energy}

We define our snake energy as

$$
E_{\text {edge }}=-\frac{1}{L} \int_{\mathbf{r}}\left|\left\langle\hat{\boldsymbol{\theta}}, \frac{\mathbf{r}^{\prime}}{\left\|\mathbf{r}^{\prime}\right\|}\right\rangle\right| \rho_{\text {opt }}(\mathbf{r}) \mathrm{d} \mathbf{r},
$$

where $\hat{\boldsymbol{\theta}}$ and $\rho_{\text {opt }}$ are the output of the steerable filter described in Section 2.2. The vector $\mathbf{r}(t)=(x(t), y(t))$, and $\mathbf{r}^{\prime}(t)=$ $\left(x^{\prime}(t), y^{\prime}(t)\right)$ are the position and derivative vectors, respectively. The first term of the integrand in (2) is the absolute value of the inner product between $\hat{\boldsymbol{\theta}}$ and a normalized version of $\mathbf{r}^{\prime}$. The absolute value of this product is large when the tangents of the contour are locally aligned with the angles of maximal filter response (see Figure 2). The second term of the integrand favors locations $\mathbf{r}$ where the steerable filter's response, and therefore $\rho_{\text {opt }}(\mathbf{r})$, is large. We normalize the line integral by the length of the curve $L$ to obtain a dimensionless energy. Finally, the energy is set with a minus sign since we define the snake optimization as a minimization process.

As a properly tuned steerable filter responds strongly to all structures of interest in the image, the magnitude of the filter response is large even at the locations where edges (or ridges) meet. The term of the energy functional that enforces $\hat{\boldsymbol{\theta}}$ and $\frac{\mathbf{r}^{\prime}}{\left\|\mathbf{r}^{\prime}\right\|}$ to have the same orientation gives additional robustness in cases where patterns of interest are in close contact. We illustrate in Figure 3 how the presence of an orientation-dependent term increases the overall robustness of the algorithm.

\section{IMPLEMENTATION DETAILS}

For the sake of efficiency, the basis functions are pre-computed and stored in lookup tables. This allows for real-time response when interacting with the anchor points and tangent vectors. Since our snake is defined by a small number of parameters ( $M$ anchor points plus $M$ tangent vectors in $2 \mathrm{D}$ ), we carry out the optimization in an efficient way relying on Powell-like line-search methods [16] which require the derivatives of the energy function with respect to the parameters (i.e., the Hermite spline coefficients) and benefit from a quadratic speed of convergence.

Compared to many other implicit and global parametric snake models, our active contour involves a smaller number of control 


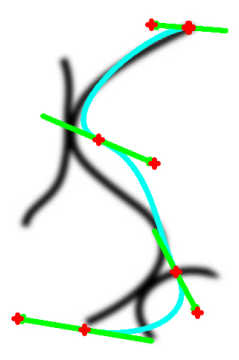

Initialization

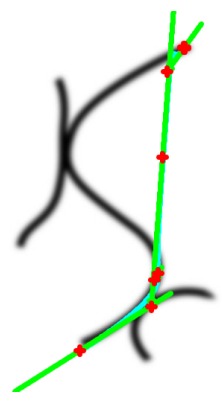

Amplitude only

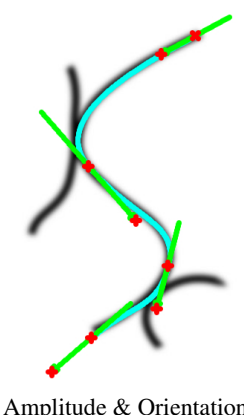

Amplitude \& Orientation
Fig. 3. Demonstration of the effect of the inner product between $\hat{\boldsymbol{\theta}}$ and $\frac{\mathbf{r}^{\prime}}{\left\|\mathbf{r}^{\prime}\right\|}$ on a synthetic image consisting of ridges. Left: initial position of the Hermite snake. Center: optimization based only on the integral of the steerable filter's response. Right: optimization according to the energy proposed in (2). Results were obtained using $N=4$ in the design of the steerable filter.

points. This eases the interaction for the end-user. We take advantage of this framework by providing an intuitive user interface through which the position and shape of the snake can be manually edited through manipulation of the control points and their tangents. As a result, our method can be deployed interactively by combining steps of snake initialization, optimization, and correction. Our snake framework has been implemented as an open-source plugin for the multi-platform software ImageJ [10]. It is made available on the Biomedical Imaging Group website ${ }^{1}$.

\section{EXPERIMENTS}

We now present two applications of our active-contour method in microscopic images. The first dataset features edge- and the second ridge-like structures. These examples illustrate the versatility of our approach through its modality-dependent initialization, automatic optimization, and user-friendly interface.

\subsection{Segmentation of Bioimages}

\subsubsection{Extraction of Nerve Fiber Layer Profile}

We processed optical coherence tomography (OCT) scans of the retina. This type of medical image is commonly used to identify and assess retinal abnormalities by measuring the thickness of the retinal nerve fiber layer (NFL). The challenge was to extract the profile of the NFL in a precise way, which appears as a possibly discontinuous and faint edge-like structure on OCT data. For the three images presented here, we set $M=4, M=5$, and $M=4$. We manually provided a rough initialization of the snake position along the NFL, and run the automatic optimization process until convergence using an edge-sensitive steerable filter with $N=3$. The results in Figure 4 demonstrate that our Hermite snake is able to accurately extract the upper profile of the NFL. The method can be used in a similar way to extract the lower profile of the NFL, thus allowing for a precise measurement of the thickness of the retinal nerve fiber layer by simply computing the distance between the two snakes at any point. The optimization process only took a few seconds in each case.

${ }^{1}$ http://bigwww.epfl.ch/algorithms/openhermitesnake/

\subsubsection{Detection of Anteroposterior Axis in C. Elegans}

We investigated the problem of implementing a semi-supervised method for outlining the morphology of the model organism $\mathrm{C}$. Elegans. We processed two types of microscopic image data. The first are images of wild-type $\mathrm{C}$. Elegans hermaphrodite with nuclear staining $^{2}$. The challenge there lies in the fact that the nuclei of all cells are visible, and the organism therefore exhibits a nonuniform gray-level pattern.

The second kind of data features C. Elegans worms expressing GFP in the GABA neurons ${ }^{3}$. These images contain less details than the previous ones, but remain challenging since many interfering ridge-like structures are still present. Yet, the proposed snake method (with $M=5, M=3$, and $M=4$, respectively) is able to accurately extract the anteroposterior axis, as shown in Figure 5. The present method allows us to capture the longitudinal line of the worm without having to detect the contour of the system. The optimization process was fast enough for interactive feedback $(3.75 \mathrm{~s}, 0.23 \mathrm{~s}$, and $2.55 \mathrm{~s}$ respectively, with $N=4$ for the design of the ridge-sensitive steerable filter).

\section{CONCLUSION}

Our contribution in this paper is a new model of open-ended snake. It is built using two complementary basis functions that jointly interpolate the contour points and their tangents. The approach is complemented with an intuitive user-interface that facilitates extensive interaction with the contour. Our model is driven by a novel energy term relying on steerable filters. As a consequence, our method can adapt to different detection problems by changing the order of the steerable filter. Our active contour model involves a few parameters only. This is advantageous in terms of memory usage and computational speed. We have demonstrated the suitability of the approach for real-life problems involving the detection of both edgeand ridge-like structures in biomedical images.

\section{REFERENCES}

[1] M. Kass, A. Witkin, and D. Terzopoulos, "Snakes: Active contour models," International Journal of Computer Vision, vol. 1, pp. 321-331, January 1988.

[2] R. Delgado-Gonzalo and M. Unser, "Spline-based framework for interactive segmentation in biomedical imaging," IRBMIngénierie et Recherche Biomédicale / BioMedical Engineering and Research, vol. 34, pp. 235-243, June 2013.

[3] T. McInerney and D. Terzopoulos, "Deformable models in medical image analysis," in Proceedings of the Workshop on Mathematical Methods in Biomedical Image Analysis, (San Francisco, CA), pp. 171-180, June 1996.

[4] A. Jain, Y. Zhong, and M.-P. Dubuisson-Jolly, "Deformable template models: A review," Signal Processing, vol. 71, pp. 109-129, December 1998.

[5] J. Montagnat, H. Delingette, and N. Ayache, "A review of deformable surfaces: Topology, geometry and deformation," Image and Vision Computing, vol. 19, pp. 1023-1040, December 2001.

\footnotetext{
${ }^{2}$ Images publicly available at http://www.genome.gov/10000570/ and http://www.plosbiology.org/article/info:doi/10.1371/journal.pbio.0030030/ (accessed September 2013).

${ }^{3}$ Courtesy of Hammarlund Lab, Yale Stem Cell Center.
} 

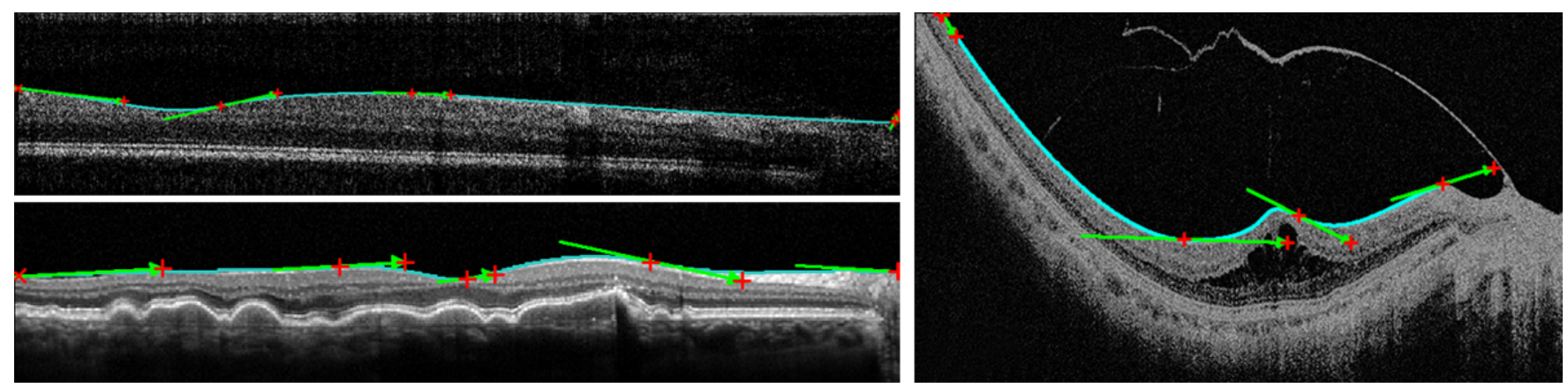

Fig. 4. Outline of the NFL profile using the Hermite snake in OCT images of the retina. Left column, top: $1077 \times 229$ (courtesy of the Institute of Applied Optics, EPFL). Left column, bottom: $570 \times 140$, and right column: $570 \times 326$ (both courtesy of the Ophthalmic Photographers' Society Inc.).
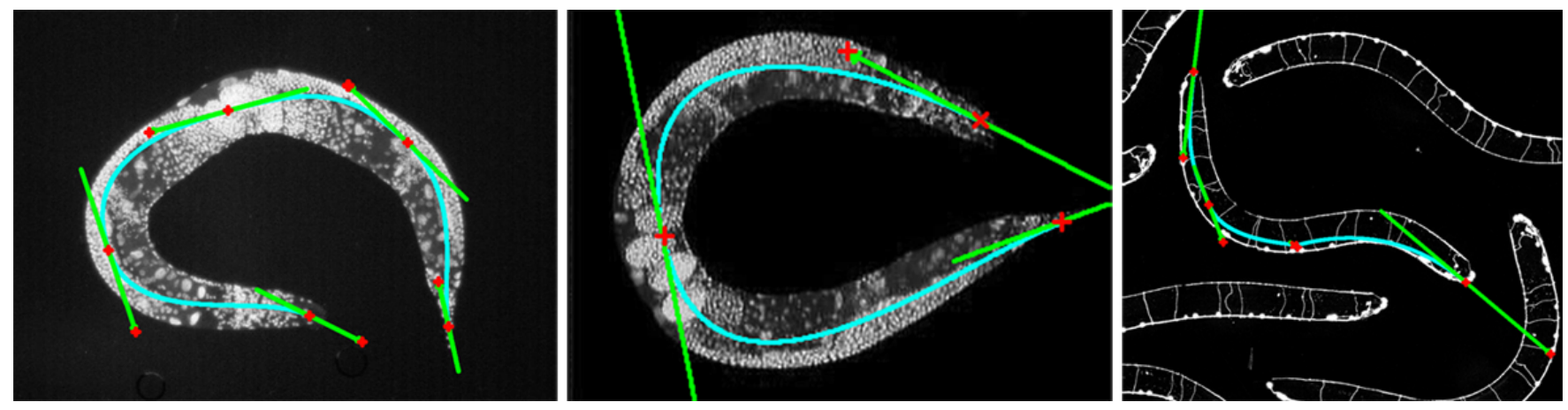

Fig. 5. Detection of the anteroposterior axis using the Hermite snake in C. Elegans with different staining methods. Left: $810 \times 588$, nuclear staining. Center: $250 \times 354$, nuclear staining. Right: $1178 \times 768$, neuron staining.

[6] M. Jacob, T. Blu, and M. Unser, "Efficient energies and algorithms for parametric snakes," IEEE Transactions on Image Processing, vol. 13, pp. 1231-1244, September 2004.

[7] L. D. Cohen and R. Kimmel, "Global minimum for active contour models: A minimal path approach," International Journal of Computer Vision, vol. 24, pp. 57-78, August 1997.

[8] P. Brigger, J. Hoeg, and M. Unser, "B-spline snakes: A flexible tool for parametric contour detection," IEEE Transactions on Image Processing, vol. 9, pp. 1484-1496, September 2000.

[9] R. Delgado-Gonzalo, P. Thévenaz, C. Seelamantula, and M. Unser, "Snakes with an ellipse-reproducing property," IEEE Transactions on Image Processing, vol. 21, pp. 12581271, March 2012.

[10] M. D. Abràmoff, P. J. Magalhães, and S. J. Ram, "Image processing with ImageJ," Biophotonics International, vol. 11, pp. 36-42, July 2004.

[11] W. Neuenschwander, P. Fua, L. Iverson, G. Székely, and O. Kübler, "Ziplock snakes," International Journal of Computer Vision, vol. 25, pp. 191-201, December 1997.

[12] Y. Wong, P. Yuen, and C. Tong, "Segmented snake for contour detection," Pattern Recognition, vol. 31, pp. 1669-1679, November 1998.

[13] W. Dahmen, B. Han, R.-Q. Jia, and A. Kunoth, "Biorthogonal multiwavelets on the interval: Cubic Hermite splines," Constructive approximation, vol. 16, pp. 221-259, February 2000.
[14] K. Jetter and G. Zimmermann, "Polynomial reproduction in subdivision," Advances in Computational Mathematics, vol. 20, pp. 67-86, January 2004.

[15] M. Jacob and M. Unser, "Design of steerable filters for feature detection using Canny-like criteria," IEEE Transactions on Pattern Analysis and Machine Intelligence, vol. 26, pp. 10071019, August 2004.

[16] W. Press, S. Teukolsky, W. Vetterling, and B. Flannery, $\mathrm{Nu}$ merical Recipes: The Art of Scientific Computing. Cambridge, UK: Cambridge University Press, 1986. Third edition, 818 p. 